\title{
An evolving function of DNA-containing exosomes in chemotherapy-induced immune response
}

\author{
Cell Research (2017) 27:722-723. doi:10.1038/cr.2017.74; published online 19 May 2017
}

Chemotherapy is a predominant strategy to treat cancer and is often associated with toxicities like severe diarrhea that puts patients at additional risk and can hinder treatment strategies. Lian et al. recently explored the immune-mediated mechanisms of Irinotecan-induced diarrhea in colorectal cancer and found that double-stranded DNA in small vesicles can launch inflammation pathways in immune cells through the cytosolic DNA sensor AIM2.

Until more targeted and generally less toxic therapies become available to treat each cancer type, chemotherapy will continue to be a standard of care. These agents are typically responsible for adverse effects in multiple organ systems due to their nonspecific action on all proliferating cells and general association with immunosuppression. Damage to constantly renewing tissues like the gut and intestine can cause the release of immunostimulatory factors that increase antitumor efficacy, but also elicit secondary conditions like diarrhea, fatigue, nausea, etc $[1,2]$. These events often limit the effectiveness of chemotherapy regimens because doses are reduced, given less frequently, or stopped completely if severe enough.

Recently published in Cell Research, Lian et al. [3] studied the underlying mechanisms of Irinotecan-induced stimulation of innate immune responses that are facilitated by double-stranded DNA (dsDNA) uptake. This increases inflammation and limits the maximal tolerated dose in patients. Irinotecan is a topoisomerase I inhibitor that is often used to treat lung and colon cancers, and has been well documented clinically to be associated with diarrhea and neutropenia [4]. In plasma samples from 69 colorectal cancer (CRC) patients collected just prior to and $48 \mathrm{~h}$ post Irinotecan administration, the authors observed an increase in dsDNA concentrations after treatment, with the most robust elevation occurring in patients with severe diarrhea. To confirm this finding, a xenograft mouse model was generated by subcutaneously injecting MC-38 murine colon carcinoma cells and subsequently administering Irinotecan into both tumor-bearing and nontumor-bearing mice. Irinotecan treatment resulted in shortening of the small intestine and sharp increases in dsDNA present in peritoneal lavage fluid in both groups, which suggests that Irinotecan is responsible for the increased release of dsDNA. Furthermore, dsDNA induced mature IL-1 $\beta$ secretion and inflammasome activation when transfected into bone marrow-derived macrophages (BMDM), which is the primary cause of side effects like diarrhea.

Given that dsDNA uptake led to mature IL- $1 \beta$ secretion, the authors assessed the role of the cytosolic dsDNA receptor AIM2, which facilitates IL-1 $\beta$ maturation in innate immune cells. Transfection of dsDNA into BMDM and dendritic cells derived from AIM2deficient mice attenuated release of IL$1 \beta$ and IL-18. This suggests that AIM2 mediates the inflammatory program that is activated when dsDNA is present in the cytosol of innate immune cells. This led the authors to question how the released dsDNA was able to enter these cells. The various mechanisms of dsDNA delivery and entry between cells as free fragments or through association with cell-secreted vesicles are being actively explored and are addressed in Figure 1.

Exosomes are 50-150 $\mathrm{nm}$ bilayered membrane vesicles that have emerged as significant contributors to intercellular communication [5]. Previous work shows that they contain dsDNA, including driver mutations that have potential value as diagnostic and prognostic biomarkers [6, 7]. Aware of this, the authors investigated whether exosomes facilitate dsDNA entry to the cytosol of macrophages and discovered that a majority of dsDNA contained in the cell culture supernatant of Irinotecan-treated cells was associated with exosomes. Furthermore, they showed a reduction of dsDNA in the culture media when cells were treated with the neutral sphingomyelinase inhibitor GW4869 that is commonly used to attenuate exosome secretion. This was further expanded on and a functional connection between exosome release and Irinotecan-induced inflammation was made in vivo by treating mice with both GW4869 and Irinotecan. This led to a reduction of dsDNA in peritoneal lavage fluid and attenuated the severity of diarrhea, body weight loss, and intestine shortening. Additionally, other in vivo experiments showed that either genetic deletion of AIM2 or treatment with the inflammasome inhibitor thalidomide reduced intestinal shortening and damage without reducing the anti-tumor effect of Irinotecan. 


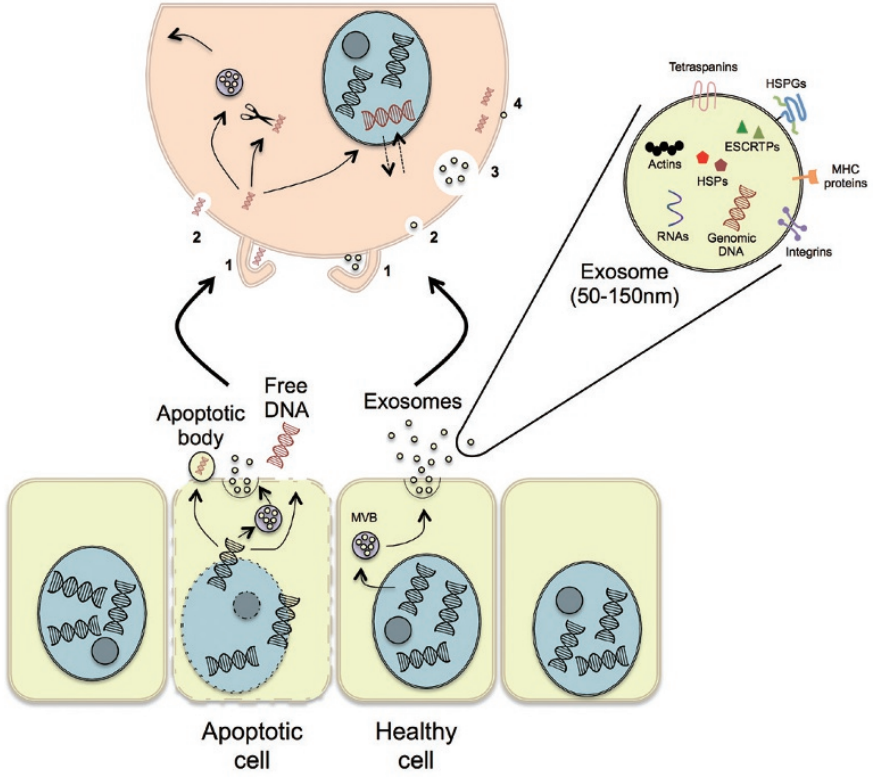

Figure 1 Routes of exogenous dsDNA delivery to other cells. A simplified overview of what is currently known about dsDNA transfer between cells. dsDNA exits apoptotic cells through apoptotic bodies $(1 \mu \mathrm{m})$, exosomes $(50-150 \mathrm{~nm})$, or as free fragments. Both healthy and cancer cells not undergoing cell death also package dsDNA in exosomes. Previous studies suggest that free dsDNA fragments enter cells primarily through 1) macropinocytosis and 2) endocytosis in a sequence-dependent manner, after which either enter the nucleus, get degraded by cytosolic nucleases, or potentially get repackaged into de novo exosomes [8-10]. Furthermore, Shimizu et al. [10] suggest that foreign dsDNA in the cytosol can enter the nucleus and visa versa and either be degraded or remain in the nucleus for more than one cell cycle. As described in a review by Mulcahy et al. [11], dsDNA-containing exosomes can enter cells through 1) macopinocytosis, 2) endocytosis (multiple mechanisms), 3) phagocytosis, and 4) membrane fusion, but the fate of dsDNA in the exosomes taken up through these mechanisms remains to be fully elucidated. Representative images are not to scale. MVB, multivesicular body; HSPGs, heperan sulfate proteoglycans; HSPs, heat shock proteins; ESCRTPs, endosomal sorting complexes required for transport proteins.

Taken together, this work bridges and advances multiple fields of study by further demonstrating the role of exosomes in intercellular communication, assigning AIM2 as the central mediator in CRC for Irinotecan-induced toxicities, and showing that ablation of either dsDNA abundance through prevention of exosome secretion or AIM2 signaling limits the severity of these toxicities without negatively impacting the efficacy of Irinotecan. This study provides some important insights that may have significant clinical impact, but many other questions also remain to be addressed. For example, the au- thors showed that a slight majority of dsDNA in cell culture supernatants was associated with exosomes after Irinotecan treatment, but what about in vivo? Furthermore, there is also a proportion of dsDNA that is not associated with microvesicles or exosomes. Does free dsDNA interact and function in dendritic cells and macrophages differently from dsDNA associated with exosomes? Mittra et al. [8] have demonstrated that fragmented dsDNA and chromatin in the blood of cancer patients can integrate into the genomes of healthy cells and activate DNA damage repair pathways, which can eventually lead to apoptosis. This begs the question: do different cell types respond to and process free dsDNA and/or encapsulated dsDNA in a similar or varied fashion? Separate from these current unknowns in our understanding of basic biology, it will be fascinating to learn whether dampening the innate immune response to chemotherapeutics like Irinotecan will have a significant impact on reducing the severity of harmful side effects.

\section{Competing Financial Interests}

MD Anderson Cancer Center and RK hold patents in the area of exosome biology and are licensed to Codiak Biosciences Inc. MD Anderson Cancer Center and RK are stock equity holders in Codiak Biosciences Inc. $\mathrm{RK}$ receives research support from $\mathrm{Co}$ diak Biosciences Inc. and serves as a member of the board of directors.

\section{Paul Kurywchak ${ }^{1}$, Raghu Kalluri ${ }^{1}$}

${ }^{I}$ Department of Cancer Biology, Metastasis Research Center, University of Texas MD Anderson Cancer Center, Houston, TX 77005, USA

Correspondence: Raghu Kalluri

E-mail: rkalluri@mdanderson.org

\section{References}

1 Bracci L, Schiavoni G, Sistigu A, et al. Cell Death Differ 2014; 21:15-25.

2 Livshits Z, Rao RB, Smith SW. Emerg Med Clin N Am 2014; 32:167-203.

3 Lian Q, Xu J, Yan S, et al. Cell Res 2017; 27:784-800.

4 Bleiberg H, Cvitkovic E. Eur J Cancer 1996; 32A Suppl 3:S18-S23.

5 Valadi H, Ekstrom K, Bossios A, et al. Nat Cell Biol 2007; 9:654-659.

6 Kahlert C, Melo SA, Protopopov A, et al. $J$ Biol Chem 2014; 289:3869-3875.

7 Yang S, Che SP, Kurywchak P, et al. Cancer Biol Ther 2017; 18:158-165.

8 Mittra I, Khare NK, Raghuram V, et al. $J$ Biosci 2015; 40:91-111.

9 Lehmann MJ, Sczakiel G. Gene Ther 2005; 12:446-451.

10 Shimizu N, Kamezaki F, Shigematsu S. Nucleic Acids Res 2005; 33:6296-6307.

11 Mulcahy LA, Pink RC, Carter DR. $J$ Extracell Vesicles 2014; 3. doi:10.3402/jev. v3.24641. 